\title{
What will physical organic chemistry achieve in the 21st century?
}

\author{
Ronald Breslow \\ Department of Chemistry, Columbia University, New York, NY 10027, USA,
}

Biography: Ronald Breslow has been doing physical organic chemistry at Columbia University since 1956. He has won a number of awards, including the ACS James Flack Norris Award for Physical Organic Chemistry and the U.S. National Medal of Science, and is the 1996 President of the American Chemical Society

In some senses physical organic chemistry is the broadest subdiscipline of chemistry. It uses all the methods of physical chemistry and a few of its own. It uses all the methods of synthetic organic chemistry to make the new molecules needed for studies. In some branches it addresses questions that were formerly the province of biochemists, while in other areas it considers the properties of solids that include the questions and methods used in material science and electronics. It is moving rapidly into the field of organometallic chemistry, with only a short leap needed into inorganic chemistry. It devises techniques for analytical chemistry, and heavily depends on analytical methods for its studies. It increasingly uses computer methods, based on quantum mechanics and on molecular mechanics, to predict and account for experimental findings.

Thus some definition of the field is important, before we consider where it is headed. The central questions addressed are mainly related to the relationship between chemical structure and chemical properties. Also, there is great interest in understanding reaction mechanisms. The methodology heavily involves the idea of a controlled experiment. That is, no explanation is considered acceptable unless comparison experiments have been done in which the important factors are varied, and it is seen that the results support (but unfortunately usually do not prove) the explanation. The major output of physical organic chemistry is a theory, an idea, an explanation. This may be a guide to new methodology, or practical applications, but the idea is the centerpiece.

We can contrast this with some other branches of chemistry. For example, in medicinal chemistry the concern is to relate chemical structure to biological properties. In synthetic organic chemistry the concern is to demonstrate either new methodology or new strategies and tactics, but the targets are often natural compounds whose properties are already known. Structural organic chemists determine the chemical nature of newly discovered species. Sometimes they then propose how those species might act or react, but in general without the controlled experiments to validate the proposals.

Physical chemists address some of the same questions that physical organic chemists address, but without the powerful tool of synthetic methods to make new compounds available. Inorganic chemists address many of the same questions that physical organic chemists address, and in organometallic chemistry the borderline between the two fields is particularly hazy. In general inorganic chemists have a better idea of how to synthesize inorganic species, and a poorer idea of how to make any organic components of their molecules, but these failings are not characteristic of the best chemists in each field.

Not all chemists stay within a prescribed area, of course. Synthetic chemists who invent a new reaction may do the physical organic chemistry needed to help understand it. Physical organic chemists may well have to develop new synthetic methodology to make target compounds of interest. Physical chemists may make an unknown compound to help their studies, and thus practice physical organic chemistry for a while. Thus in describing the future of physical organic chemistry we must remember that some parts of it may be worked on by biochemists, by synthetic organic chemists, or even by electrical engineers with a molecular bent. 


\section{SOME GOALS FOR THE FUTURE}

It is easy to think of what we would like to be able to do that is now out of our reach. A particularly clear example is in the field of reaction mechanisms. Currently we deduce likely mechanisms from indirect evidence - kinetic dependences, medium effects, temperature effects, structure-reactivity relationships, etc. We really need methods that will allow us to observe reactions directly on the extremely short timescale over which they occur. In a few simple cases this is now possible with fast laser methods (1).

My own prejudice, shared by many others, is that the true future of the mechanism field involves computation, validated by experiment. We would really like to see a movie of the reaction, with all the velocities and energies, and a computer can generate this now. The problem is that we don't know whether the picture is correct. For example, we have proposed a mechanism for the enzyme ribonuclease (2), and at least one computer calculation supports our new mechanism (3). Is that calculation correct? If so, the problem is solved.

In general, I believe that we will be able to describe the mechanisms of enzymatic reactions in detail, and more important we will understand why they are so effective. We will also be able to imitate them with synthetic catalysts that incorporate the same features. This, which I have called biomimetic chemistry (4), will greatly increase the ability of chemists to perform reactions with high selectivity and efficiency, and with minimal energy requirements.

Physical organic chemistry will also let us do what I call Nature Appreciation: understanding the chemical reason for some of the biological structures we find. For instance, we have done such a study for the vitamin thiamin (5), seeing how and why variants of the natural structure are inferior in the chemical properties that are relevant to the biological catalysis performed by thiamin pyrophosphate. We $(6)$ and others $(7,8,9)$ have also done this kind of study for nucleic acids by seeing how the natural compounds excel in their ability to form double helical structures relative to some variants that could have been formed under primitive earth conditions. Work on micelles and vesicles (10) has a number of goals, but one is to let us understand the chemistry of living cells and their membranes.

Another general goal is to extend the understanding we have and will have about simple purely organic chemistry to the fields of organometallic and inorganic chemistry. Some far-seeing chemists are already active in this field $(11,12)$. We also need to learn how the electrical and optical properties of materials can be affected and controlled by appropriate structural chemistry, a field that is also actively moving (13).

We also need to understand the properties of unusual unstable chemical species, not just normal molecules. Our work on antiaromatic molecules was an example (14), and with new physical methods many reactive intermediates are now well characterized, such as carbenium ions (15). Some reactive intermediates, in particular carbenes, are major components of interstellar gas clouds, whose collapse leads to the birth of stars. In the study of such species, physical organic chemistry makes a contribution to astronomy. Physical organic chemistry also furnishes the tools by which prebiotic chemistry is investigated, furnishing clues as to how life itself may have originated under conditions likely in a primitive earth, or elsewhere in the universe.

There are some practical fields in which physical organic chemistry will continue to make important contributions. In clinical chemistry, for instance, physical organic chemists are the ones who will translate a molecular binding event into a detectable signal so that tiny amounts of biologically important compounds can be detected and quantified. Such analytical methods are an important application of the general field of Molecular Recognition.

Physical organic chemists will learn how to better catalyze industrial processes, and how to make polymers with well defined structures and properties. Physical organic chemists will learn how to design materials, such as refrigerants, that have a lifetime sufficient for their use but then degrade so they are not environmentally persistent. Physical organic chemists will design the catalysts and methods to convert methane into higher hydrocarbons and other useful substances.

These are some of the challenges, and likely advances, that I can predict, but there is one more prediction I can make with certainty. Smart creative physical organic chemists will come up with advances and inventions that are anticipated by none of the chapters in this volume, including this one, and we will all say: why didn't we think of that? 


\section{REFERENCES}

1. J. C. Polanyi and A. H. Zewail. Acc. Chem. Res. 28, 119 (1995).

2. R. Breslow. Acc. Chem. Res. 24, 317 (1991).

3. B. Wladkowski, M. Krauss and W. Stevens. J. Am. Chem. Soc. 117, 10537 (1995).

4. R. Breslow. Acc. Chem. Res. 28, 146 (1995).

5. R. Breslow and E. McNelis. J. Am. Chem. Soc. 81, 3080 (1959).

6. R. Jin, W. H. Chapman, Jr., A. R. Srinivasan, W. K. Olson, R. Breslow and K. Breslauer. Proc. Natl. A A ad. Sci. U.S.A. 90, 10568 (1993).

7. A. Eschenmoser and E. Loewenthal. Chem. Soc. Rev. 21, 1 (1992).

8. K.-Y. Jung and C. Switzer. J. Am. Chem. Soc. 116, 6059 (1994).

9. R. Kierzek, L. He and D. H. Turner. Nucleic Acids Res. 20, 1685 (1992).

10. e.g., R. A. Moss, G. Li and J.-M. Li. J. Am. Chem. Soc. 116, 805 (1994); N. J. Turro, J. K. Barton and D. A. Tomalia. Acc. Chem. Res. 24, 332 (1991); F. C. Meldrum, N. A. Notov and J. H. Fendler. J. Phys. Chem. 98, 4506 (1994); F. M. Menger. Acc. Chem. Res. 26, 206 (1993).

11. e.g., R. Bergmann. Acc. Chem. Res. 28, 154 (1995).

12. e.g., R. H. Grubbs and G. W. Coates. Acc. Chem. Res. 29, 85 (1996).

13. e.g., Q. Zhou and T. M. Swager. J. Am. Chem. Soc. 117, 12593 (1995).

14. R. Breslow. Acc. Chem. Res. 6, 393 (1973).

15. e.g., G. A. Olah. Science 270, 1417 (1995). 